\title{
On the Crystal Structure of the S-phase Precipitate in Al-Cu-Mg Alloy
}

\author{
Hwang Su Kim ${ }^{*}$ and Simon P. Ringer ${ }^{* *}$ \\ * Department of Physics, Kyungsung University, Namku, Busan 608-736, South Korea \\ **Australian Key Centre for Microscopy \& Microanalysis, The University of Sydney, NSW 2006, \\ Australia
}

The S-phase $\left(\mathrm{Al}_{2} \mathrm{CuMg}\right)$ occurs over a wide range of scientifically interesting and technologically significant 2xxx Al-Cu-Mg alloys. This equilibrium phase occurs as fine lath-shaped precipitates that grow with a crystallographic habit plane parallel or near to $\{210\}_{\mathrm{Al}}$. The tendency for nucleation of this phase during thermomechanical processing of these alloys is an important consideration in the development of structure-property relationships. For this reason, it is important to understand the crystal structure of precipitate phases as accurately as possible so that a detailed understanding of their nucleation and growth can be developed. In this work, we revisit the structure of the $S$ phase by performing new electron diffraction experiments and simulations that allow us to assess recent proposals for the structural model.

Perlitz and Westgren (PW) first derived the crystal structure of the S-phase using X-ray diffraction from a single crystal [1]. Recently, Radmilovic et al. (RaVel) used high resolution transmission electron microscopy (HRTEM) to propose a revision of the structure of the S-phase precipitate in Al-Cu-Mg alloys [2,3]. The structure of the RaVel model is very similar to that of the PW model, though it does exchange certain $\mathrm{Cu}$ and $\mathrm{Mg}$ atom positions. Moreover, Wolverton recently reported a fist-principles total energy calculation for the S-phase structure that was consistent with the PW model and not consistent with the RaVel model [4]. Furthermore, Alekseyev et al. used careful Xray diffraction experiments to precipitates in a single crystal of $\mathrm{Al}-1.2 \mathrm{Cu}-1.2 \mathrm{Mg}$ (at. \%) alloy [5]. Figure 1(b) of that paper reveals strong $(020)_{\mathrm{s}}$ reflections.

Here, we report the result of a calculation of the ratio of the structure factor square (SFS) for $(020)_{\mathrm{s}}$ $\mathrm{X}$-ray scattering for the PW and RaVel models as 48:0.5. This assessment favours the PW model and is inconsistent with the RaVel model. Our X-ray diffraction pattern (XRDP) simulation for the photograph or Kforogram, based on SFS calculations, is clearly supportive of the PW model. We have also performed electron diffraction experiments using a JEOL 3000F \& 2010 TEM on an Al1.1Cu-1.7Mg (at. \%) alloy following solution treatment $\left(525^{\circ} \mathrm{C}, 1 \mathrm{~h}\right)$, water quenching and ageing for $500 \mathrm{~h}$ at $150{ }^{\circ} \mathrm{C}$ (peak hardness). We have compared the observed diffraction intensity to the SFS for electron scattering to evaluate the above two crystal structural models for S-phase models

Figure 1(a) is a $\langle 112\rangle_{\mathrm{Al}}$ bright field image and reveals numerous fine lath-shaped S-phase precipitates and Fig. 1(b) is the corresponding selected area electron diffraction (SAED) pattern. We also provide SAED simulations of S phase precipitation in Al using both the RaVel and PW models, respectively, in Figs. 1(c) and (d). We have used the formulation in [6] and assumed that the size of the precipitates is so very fine that the kinematical electron diffraction treatment is valid. Table 1 summarises our qualitative estimations of the strong (s), medium (m) and weak (w) diffracted intensity for S-phase reflections as observed in the experimental SAED's. The calculated SFS data for electron scattering from the two structural models is also included in this table. In the simulated SAED's, an S-phase precipitate size of $(30 \sim 100) \mathrm{a}_{\mathrm{s}} \times(3 \sim 5) \mathrm{b}_{\mathrm{s}} \times(3 \sim 5) \mathrm{c}_{\mathrm{s}}\left(\mathrm{a}_{\mathrm{s}}=0.405, \mathrm{~b}_{\mathrm{s}}=0.923, \mathrm{c}_{\mathrm{s}}=0.713\right.$ $\mathrm{nm}$ ) was used, on the basis of our observations. Our results are more consistent with PW structural model than for the RaVel model. 


\section{References}

[1] H. Perlitz and A. Westgren, Arkiv. Kemi. Mineral. Geol.,1943, B16, 13.

[2] V. Radmilovic, R. Kilaas, U. Dahmen and G. J. Shiflet, Acta Mater. 47 (1999) 3987-3997.

[3] R. Kilaas and V. Radmilovic, Ultramicroscopy 88 (2001) 63-72.

[4] C. Wolverton, Acta Mater. 49 (2001) 3129-42).

[5] A.A. Alekseyev, L.B. Ber, L. G. Klimovich and O. S. Korobov, Phys. Met. Metall. 46 (1979) 80.

[6] P.B. Hirsch, A. Howie, R.H. Nicholson, D.W. Pashley, and M.J. Whelan, Electron Microscopy of Thin Crystals, Krieger Publ., Huntington (N.Y.) 1977, p. 97.

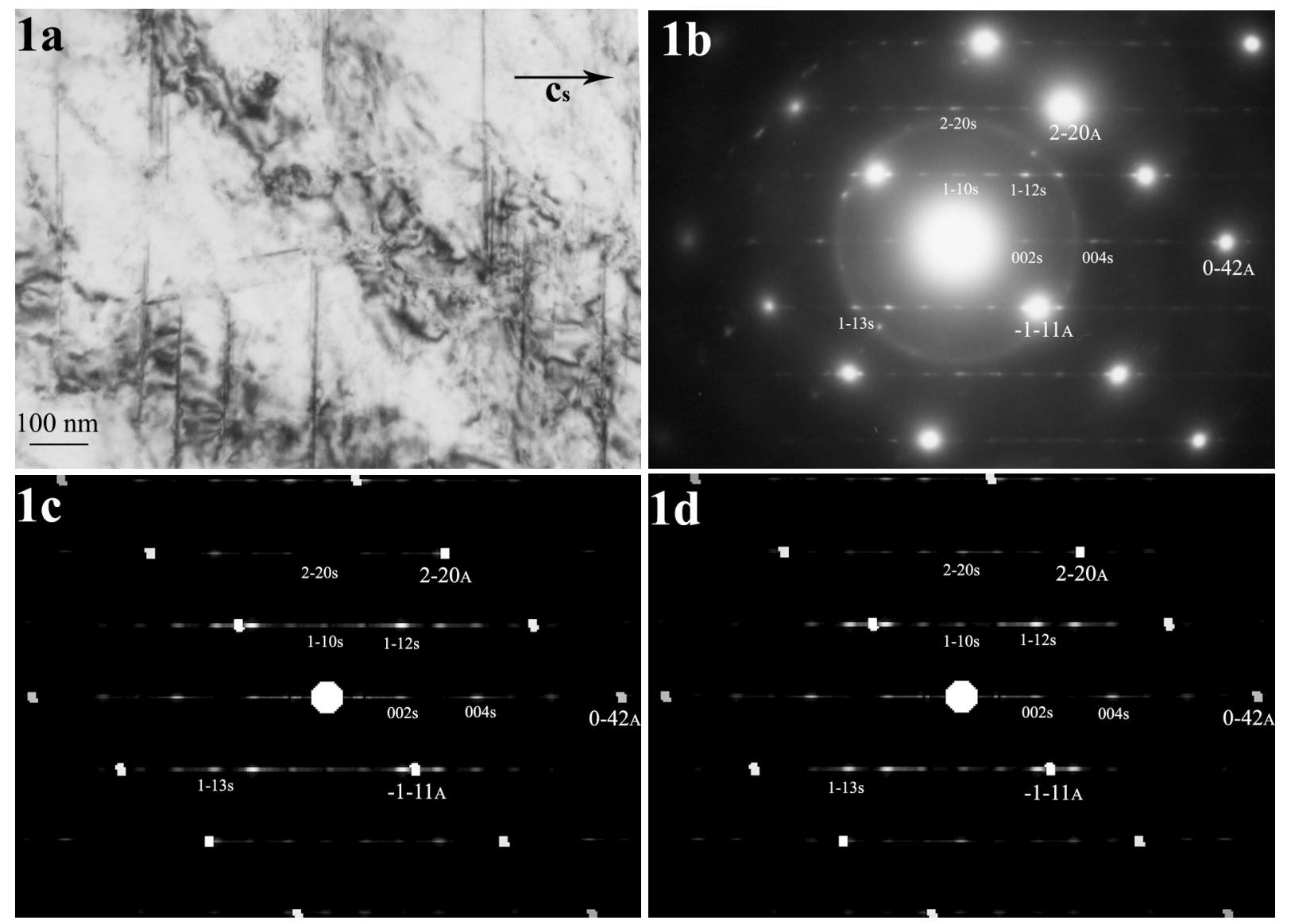

Fig. 1. BF TEM image (a) and corresponding SAED (b) for S-phase precipitates in Al with orientation $[112]_{\mathrm{Al}} \|[110]_{\mathrm{S}}$. (c) and (d) are the corresponding simulated SAED's for the RaVel and the PW models, respectively. The correspondence between the experimental and calculated diffraction intensity for reflections $(1 \overline{1} 3)$ s and $(2 \overline{2} 0)$ s is superior with the PW model.

Table 1. The observed intensity $\mathrm{I}_{\mathrm{ob}}$ for hkl reflections: $\mathrm{s}^{+}, \mathrm{s}, \mathrm{s}^{-}, \mathrm{m}+, \mathrm{m}, \mathrm{m}-, \mathrm{w}^{+}, \mathrm{w}$, and $\mathrm{w}^{-}$. The calculated values of the SFS, $|\mathrm{F}(\mathrm{hkl}) / \mathrm{F}(200)|^{2} \times 100$, are listed for the PW and the RaVel models.

\begin{tabular}{|l|c|c|c|l|c|c|c|l|l|l|c|}
\hline $\mathrm{hkl}$ & $\mathrm{I}_{\mathrm{ob} .}$ & $\mathrm{PW}$ & RaVel & $\mathrm{hkl}$ & $\mathrm{I}_{\mathrm{ob}}$ & $\mathrm{PW}$ & $\mathrm{RaVel}$ & $\mathrm{hkl}$ & $\mathrm{I}_{\text {ob. }}$ & $\mathrm{PW}$ & RaVel \\
\hline 002 & $\mathrm{w}+$ & 5.6 & 5.5 & $060^{*}$ & - & 0.6 & 6.2 & 114 & $\mathrm{~W}-$ & 2.4 & 6.1 \\
\hline 004 & $\mathrm{~s}-$ & 31.7 & 30.6 & 110 & $\mathrm{~m}$ & 1.3 & 0.4 & $220^{*}$ & $\mathrm{~m}+$ & 9.1 & 0.1 \\
\hline 006 & $\mathrm{~W}$ & 18.0 & 17.2 & 111 & $\mathrm{~m}$ & 1.6 & 0.9 & 221 & $\mathrm{~m}-$ & 1.1 & 1.1 \\
\hline $020^{*}$ & $\mathrm{~s}$ & 27.2 & 7.4 & 112 & $\mathrm{~s}+$ & 35.8 & 49.5 & 222 & $\mathrm{~W}$ & 1.8 & 0.5 \\
\hline $040^{*}$ & $\mathrm{w}$ & 5.0 & 16.4 & $113^{*}$ & $\mathrm{~s}$ & 32.8 & 10.2 & $223^{*}$ & $\mathrm{~m}$ & 5.8 & 18.2 \\
\hline
\end{tabular}

